\title{
CORRECTION
}

\section{Correction: Brain function during stages of working memory in schizophrenia and psychotic bipolar disorder}

Anna S. Huang ${ }^{1}$, Baxter P. Rogers ${ }^{2}$, Alan Anticevic ${ }^{3}$, Jennifer Urbano Blackford ${ }^{1,4}$, Stephan Heckers ${ }^{1}$ and Neil D. Woodward ${ }^{1}$

Neuropsychopharmacology (2019) 44:2143; https://doi.org/10.1038/s41386-019-0488-3

Correction to: Neuropsychopharmacology https://doi.org/10.1038/ s41386-019-0434-4, published online 11 June 2019

The original version of this Article was updated shortly after publication to correct all references of numbers (such as 2167, $2168,2165,2162$ etc.) as seen in Table 1 so as to include a comma after the 2. The comma was placed there to indicate two separate groups of numbers, 2 and 168 etc., rather than indicating a large number.

Below are the exact locations of the changes needed, and the numbers listed need a comma after the " 2 " to indicate two separate groups of numbers rather than a large number:

Table 1-all 4-digit numbers within the 'df' column

Under the Results section-Accuracy (calculated as d') did not differ between groups $(F(2167)=1.603, p=.204)$. For median reaction time (RT), main effects of condition $(F(2167)=5.962$, $p=.003 ; \mathrm{WM}>$ Control trials), and group $(\mathrm{F}(2167)=141.431$, $p<.001)$ were detected. The group $\mathrm{x}$ condition interaction was not significant $(\mathrm{F}(2167)=0.135, p=.874)$.

Encoding. A one-way ANOVA revealed significant group effects in the FEF $(\mathrm{F}(2168)=8.160, p<.001)$, SPL $(\mathrm{F}(2168)=12.088$, $p<.001)$ and IPS $(\mathrm{F}(2168)=8.758, p<.001)$

During the late delay period, the FEF and IPS showed significant differences between groups $(\mathrm{F}(2168)=10.721, \quad p<.001$ and $F(2168)=6.721$

Legend for Figure 1-There was a significant difference in reaction time for both WM $(F(2168)=3.55, p<.05)$ and $C T L$ conditions $(\mathrm{F}(2168)=5.01, p<.01)$.

This has now been corrected in both the PDF and HTML versions of the Article.

${ }^{1}$ Department of Psychiatry and Behavioral Sciences, Vanderbilt University Medical Center, 1601 23rd Ave. S., Nashville, TN 37212, USA; ${ }^{2}$ anderbilt University Institute of Imaging Sciences, Nashville, TN 37212, USA; ${ }^{3}$ Department of Psychiatry, Yale University School of Medicine, New Haven, CT, USA and ${ }^{4}$ Department of Veterans Affairs Medical Center, Research Health Scientist, Research and Development, Nashville, TN, USA

Correspondence: Anna S. Huang (anna.s.huang@vumc.org)

Published online: 2 September 2019 